\title{
Expanding the class of conditionally exactly solvable potentials
}

\author{
A. de Souza Dutra \\ Universidade Estadual Paulista-Campus de Guaratinguetá-DFQ, Avenida Dr. Ariberto Pereira da Cunha, 333, \\ Código de Endereçamento Postal 12500-000, Guaratinguetá, São Paulo, Brazil \\ Frank Uwe Girlich \\ Fachbereich Physik, Universität Leipzig, Augustusplatz 10, 04109 Leipzig, Germany
}

(Received 30 December 1993)

\begin{abstract}
Recently a class of quantum-mechanical potentials was presented that is characterized by the fact that they are exactly solvable only when some of their parameters are fixed to a convenient value, so they were christened as conditionally exactly solvable potentials. Here we intend to expand this class by introducing examples in two dimensions. As a byproduct of our search, we found also another exactly solvable potential.

PACS number(s): 03.65.Ca, 03.65.Ge, 02.90.+p
\end{abstract}

Recently the existence of a whole class of exactly solvable potentials in quantum mechanics was discovered [1]. Furthermore it was observed that these potentials obey a supersymmetric algebra [2]. In fact the potentials discussed in Ref. [1] were also discussed independently in a previous work of Stillinger [3]. In that article the author did not perceive that such potentials were only the first representatives of an entire new class of potentials. What distinguishes these potentials from the usual exactly solvable potentials is the fact that at least one of their parameters must be fixed to a specific value, because if you do not do that the potentials no longer have exact solutions. On the other hand, they cannot be classified among the so called quasi exactly solvable potentials [4-10], because these have only a finite number of exactly solvable energy levels, and this happens only when the potential parameters obey some constraint equations, so that only some of the parameters are free, and the others are fixed in terms of them. The continuous interest in obtaining and classifying quantum-mechanical potentials is because of their importance as a basis for expanding more realistic cases, and also for use of their solutions to test numerical calculations. Another possibility is that of trying to use them as a kind of theoretical laboratory, searching for new interesting physical features. Some of the examples that we will study below have, in fact, the interesting characteristic of representing a kind of semi-infinite charged string, because, as can be seen in Fig. 1, the potential is infinitely attractive along the $x$ semiaxis. Here in this work we intend to expand the class of conditionally exactly solvable (CES) potentials. This is going to be done through the presentation of two examples in two dimensions. As a byproduct of our search for CES potentials, we found another exactly solvable potential, whose solution will also be presented.

The potentials that will be considered here are

$$
\begin{aligned}
V_{\mathrm{I}}(x, y)=\left[\frac{1}{x^{2}+y^{2}}\right][ & \frac{A}{\arctan (y / x)}+\frac{B}{[\arctan (y / x)]^{1 / 2}} \\
& \left.+\frac{G_{0}}{[\arctan (y / x)]^{2}}+C\right] \\
& +D\left(x^{2}+y^{2}\right)
\end{aligned}
$$

and

$$
\begin{aligned}
& V_{\mathrm{II}}(x, y)= {\left[\frac{1}{x^{2}+y^{2}}\right] } \\
& \times\left[A\left[\arctan \frac{y}{x}\right]^{2 / 3}+\frac{B}{[\arctan (y / x)]^{2 / 3}}\right. \\
&\left.+\frac{g_{0}}{[\arctan (y / x)]^{2}}+C\right]
\end{aligned}
$$

where the function $\arctan (y / x)$ is defined in the principal branch. It can be observed that the first case above has an exact solution for any value of $G_{0}$ if $B=0$. Otherwise the case $B \neq 0$ has an exact solution for $G_{0}=-3 \hbar^{2} / 32 \mu$, and only for a restricted region of the space ( $x$ and $y$ both bigger or both less than zero).

The second potential has exact solutions only when $g_{0}=-5 \hbar^{2} / 32 \mu$. In this case the solution is valid along all the $x-y$ plane. It is not difficult to verify that these potentials are singular along the positive semiaxis $x$, as can be seen from Fig. 1. So they represent a kind of "charged string."

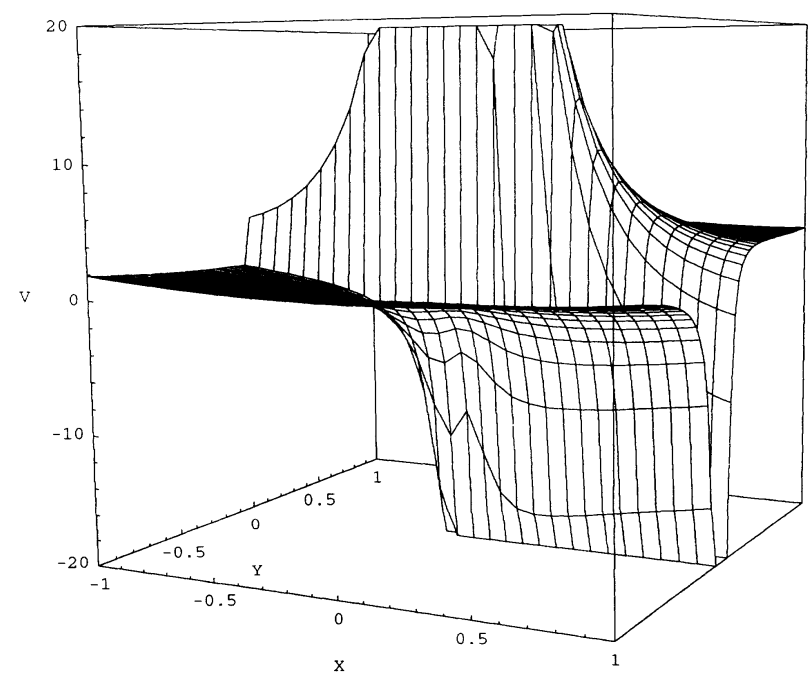

FIG. 1. Plot of the potential CES II with unitary parameters.

(c) 1994 The American Physical Society 
The solution for these potentials can be obtained through a suitable change of coordinates:

$$
x=u \cos (v), \quad y=u \sin (v) .
$$

After this change of variables, and their separation, the Schrödinger equation can be split into two others. In the variable $u$ we have that

$$
\left\{-\frac{\hbar^{2}}{2 \mu} \frac{d^{2}}{d u^{2}}+V_{e}(u)-\mathscr{E}\right\} \Phi(u)=0,
$$

where

$$
V_{e}(u)=D u^{2}+\frac{\left(C+K-\hbar^{2} / 8 \mu\right)}{u^{2}},
$$

with $K$ being the separation constant and $\mu$ the mass of the particle. The subscript $e$ in the potential stands for its exact solvability. In the variable $v$ we have

$$
\left\{-\frac{\hbar^{2}}{2 \mu} \frac{d^{2}}{d v^{2}}+V_{\mathrm{CES}}(v)-K\right\} \chi(v)=0,
$$

with $V_{\mathrm{CES}}(v)$ having two possibilities:

$$
V_{\mathrm{CES}}^{\mathrm{I}}(v)=\frac{A}{v}+\frac{B}{v^{1 / 2}}+\frac{G_{0}}{v^{2}},
$$

and

$$
V_{\mathrm{CES}}^{\mathrm{II}}(v)=A v^{2 / 3}+\frac{B}{v^{2 / 3}}+\frac{g_{0}}{v^{2}} .
$$

The wave function can be obtained by returning $\Psi(u, v)=u^{-1 / 2} \Phi(u) \chi(v)$ to the original variables.

At this point, we shall observe that the transformation used leads us to a mapping into an angle variable $v=\arctan (y / x)$, which can, in principle, create problems like multivaluedness of the wave function. However, one can see that this transformation is typical of a stereographic mapping, in this case of $\mathbb{R}^{2}$ onto $\mathrm{S}^{2}$. This kind of compactification can be done provided that one has suitable asymptotic conditions [11]. In fact, this implies that one will have a finite wave function throughout variation of the variables, and particularly that at infinity it should vanish. This imposes the condition that the parameter $D$ be nonzero, because along the positive $x$ semiaxis the wave function will be singular at infinity if $D=0$. Furthermore, there is no periodicity of the wave function in the variables $x$ and $y$, as we will see later.

So we see that the original potentials were, in some cases, reduced to one-dimensional CES potentials, whose solutions were obtained previously. Now we proceed to the solution of the above mentioned possibilities.

Case I for $B=0$ and $G_{0}$ arbitrary. The first example is that of an exactly solvable potential. In this case the potential $V_{\mathrm{I}}(x, y)$ is mapped into a harmonic oscillator with centrifugal barrier in the variable $u$, and a Coulomb plus centrifugal barrier in the variable $v$. So we only need to identify the corresponding parameters, substitute them in the equation for the "energy," which in the transformed equation is the parameter $K$, and solve it for the original energy

$$
\begin{aligned}
\delta_{n, m}^{1}(B=0)= & \left|4 C D-\frac{8 \mu A^{2} D}{\hbar^{2}\left[2 m+1+a_{1}\right]^{2}}\right|^{1 / 2} \\
& +\hbar\left(\frac{2 D}{\mu}\right)^{1 / 2}(2 n+1)
\end{aligned}
$$

where $a_{1} \equiv\left(1+8 \mu G_{0} / \hbar^{2}\right)^{1 / 2}$. It can be seen that the above spectrum is a kind of mixing of the Coulomb and harmonic oscillator spectra. The normalized wave functions are

$$
\begin{aligned}
\psi_{n, m}^{\mathrm{I}, B=0}(x, y)= & {\left[\left.\frac{32 \mu D}{\hbar^{2}}\right|^{1 / 8} \mid \frac{2 \Gamma(n+1)}{\Gamma\left(a_{1}+n+1\right)}\right]^{1 / 2}\left[\left.\left|\frac{2 \mu D}{\hbar^{2}}\right|^{1 / 2}\left(x^{2}+y^{2}\right)\right|^{a_{1} / 2}\left|\arctan \frac{y}{x}\right|^{1 / 4}\right.} \\
& \times\left.\left[\frac{\Gamma(m+1)}{\Gamma\left(a_{1}+m+1\right)}\right]^{1 / 2}\left[\left|\frac{-8 \mu K}{\hbar^{2}}\right|^{1 / 2} \arctan \frac{y}{x}\right]^{\left(2 a_{1}+1 / / 4\right.} L_{n}^{a_{1}}|| \frac{2 \mu D}{\hbar^{2}}\right|^{1 / 2}\left(x^{2}+y^{2}\right) \mid \\
& \left.\times L_{m}^{a_{1}}\left[\mid \frac{-8 \mu K}{\hbar^{2}}\right]^{1 / 2} \arctan \frac{y}{x}\right] \exp \left\{-\left|\frac{\mu D}{2 \hbar^{2}}\right|^{1 / 2}\left(x^{2}+y^{2}\right)-\left(\left.\frac{-2 \mu K}{\hbar^{2}}\right|^{1 / 2} \arctan \frac{y}{x}\right\},\right.
\end{aligned}
$$

where

$$
\begin{aligned}
& a_{2} \equiv\left(\frac{\mu}{2 \hbar^{2} D}\right]^{1 / 2} \mathscr{E}_{n, m}^{\mathbf{I}}-(2 n+1), \\
& K \equiv \frac{1}{4 C}\left[\mathscr{E}_{n, m}^{\mathbf{I}}-\frac{\hbar}{\mu}\left[\frac{2 D}{\mu}\right]^{1 / 2}(2 n+1)\right]^{2}-C,
\end{aligned}
$$

and $L_{n}^{a}(x, y)$ is the generalized Laguerre polynomial. It is remarkable that, as can be seen from Eq. (5), the domain of validity of the parameter $C$ is defined through the equation

$$
C \geq \frac{2 \mu A^{2}}{\left(2 m+a_{1}+1\right) \hbar^{2}},
$$

in order to keep the energies real. So, as the left hand side of the above equation has its minimum value when $m=0$ ( $n$ arbitrary), we must have $C \geq 2 \mu A^{2} /\left(a_{1}+1\right) \hbar^{2}$. Apparently if $C$ is less than this we should impose the condition that

$$
m \geq \operatorname{Int}\left[\frac{\mu A^{2}}{\hbar^{2} C}-\frac{1}{2}\left(a_{1}+1\right)\right],
$$

where Int( ) stands for taking the first integer greater than or equal to the argument. This is a strange feature because, in this case, the ground state would have nodes looking like an excited state. This characteristic should 
be verified through numerical calculation. Perhaps we would need some kind of analytical continuation for the energy in such cases. Now, the next case.

Case I for $B \neq 0$ and $G_{0}=-3 \hbar^{2} / 32 \mu$. In this case we have our first example of a two-dimensional CES potential. After repeating the previous procedure we get the following equation for the energy eigenstates:

$\hbar^{2}\left(m+\frac{1}{2}\right) K_{m}^{3}+2 \mu A^{2} K_{m}^{2}+\mu A B^{2} K_{m}+\frac{\mu B^{4}}{8}=0$,

with

$$
K_{m}=\frac{1}{4 D}\left[\mathscr{E}_{n, m}^{\mathrm{I}}-\left[\frac{2 \hbar^{2} D}{\mu}\right]^{1 / 2}(2 n+1)\right]^{2}-C .
$$

As we are looking for $\mathscr{E}_{n, m}^{\mathrm{I}}$, we see that it is necessary to solve a third order polynomial equation for $K_{m}$, and then use this solution in the expression for the energy

$$
\mathscr{E}_{n, m}^{\mathrm{I}}= \pm 2 \sqrt{D}\left(K_{m}+C\right)^{1 / 2}+\left(\frac{2 \hbar^{2} D}{\mu}\right)^{1 / 2}(2 n+1) ;
$$

once more we have a limitation on the parameter $C$. The solution of the equation for the parameter $K_{m}$ is obtained following the procedure appearing in Ref. [1]. This gives us

$$
K_{m}=(R+D)^{1 / 3}+(R-D)^{1 / 3}-\frac{a_{1}}{3},
$$

with $\quad D \equiv \sqrt{Q^{3}+R^{2}}, \quad Q \equiv\left(3 a_{2}-a_{1}^{2}\right) / 9, \quad R \equiv\left(9 a_{1} a_{2}\right.$ $\left.-27 a_{3}-2 a_{1}^{3}\right) / 54$, and

$$
\begin{aligned}
& a_{1} \equiv \frac{2 \mu A^{2}}{\hbar^{2}\left(m+\frac{1}{2}\right)^{2}}, \quad a_{2} \equiv \frac{\mu A B^{2}}{\hbar^{2}\left(m+\frac{1}{2}\right)^{2}}, \\
& a_{3} \equiv \frac{\mu B^{4}}{8 \hbar^{2}\left(m+\frac{1}{2}\right)^{2}} .
\end{aligned}
$$

The wave function is given by

$$
\begin{aligned}
\psi_{n, m}^{\mathrm{I}}(x, y)= & {\left[\frac{32 \mu D}{\hbar^{2}}\right]^{1 / 8}\left[\frac{\Gamma(n+1)}{2^{m+1} m ! \Gamma(a+n+1)}\right]^{1 / 2}\left[\left(\frac{2 \mu D}{\hbar^{2}}\right]^{1 / 2}\left(x^{2}+y^{2}\right)\right]^{a / 2}\left[\frac{\beta^{2}}{\hbar} \arctan \frac{y}{x}\right]^{1 / 4} } \\
& \times L_{n}^{a}\left[\left[\frac{2 \mu D}{\hbar^{2}}\right]^{1 / 2}\left(x^{2}+y^{2}\right)\right] H_{m}\left[\beta\left[\sqrt{\arctan (y / x)}-\frac{B}{2 K_{m}}\right]\right] \\
& \times \exp \left\{-\left(\frac{\mu D}{2 \hbar^{2}}\right]^{1 / 2}\left(x^{2}+y^{2}\right)-\frac{\beta^{2}}{2}\left[\sqrt{\arctan (y / x)}-\frac{B}{2 K_{m}}\right]^{2}\right\},
\end{aligned}
$$

where we defined

$$
a \equiv\left(\frac{\mu}{2 \hbar^{2} D}\right)^{1 / 2} \mathscr{E}_{n, m}^{\mathrm{I}}-(2 n+1),
$$

and

$$
\beta \equiv\left(\frac{8 \mu K_{m}}{\hbar^{2}}\right)^{1 / 4},
$$

and $H_{m}(x, y)$ is the Hermite polynomial.

It is easy to verify that the wave function, being proportional to $\arctan (y / x)$, vanishes along the positive $x$ semiaxis. This should be expected due to the singularity of the potential in this region. Moreover, it is easy to see from the wave function (14) that, as observed previously when we presented the potential, one must restrict the region of space such that $x$ and $y$ are both positive or both negative. Furthermore, the inverse tangent function is defined in the principal branch, as said previously. Finally we treat the second case.

Case II $\left(g_{0}=-5 \hbar^{2} / 72 \mu\right)$. Now we present the last example of a two-dimensional CES potential that will be discussed in this work. If we repeat once more the same procedure we get the following equation for the energy in this last case:

$$
\begin{aligned}
\pm \frac{4}{3} \sqrt{A} & {\left[(m+1 / 2) \hbar\left(\frac{9 A}{2 \mu}\right)^{1 / 2}+\frac{9 B}{4}\right]^{1 / 2} } \\
= & \frac{1}{4 C}\left[\mathscr{E}_{n, m}^{\mathrm{II}}-\left(\frac{2 \hbar^{2} D}{\mu}\right)^{1 / 2}(2 n+1)\right]^{2}-C,
\end{aligned}
$$

with the solution

$$
\begin{aligned}
\mathscr{E}_{n, m}^{\text {II }}= & \left\{ \pm \frac{16}{3} D \sqrt{A}\left[m+\frac{1}{2}\left(\frac{9 \hbar^{2} A}{2 \mu}\right]^{1 / 2}+\frac{9 B}{4}\right]^{1 / 2}\right. \\
& +4 C D\}^{1 / 2}+\left(\frac{2 \hbar^{2} D}{\mu}\right]^{1 / 2}(2 n+1)
\end{aligned}
$$

This time we need to do a careful analysis of the range of validity for the potential parameters. In the case of the parameter $B$, we have the restriction

$$
B \geq-\left(\frac{2 \hbar^{2} A}{9 \mu}\right)^{1 / 2},
$$

in order to include $m=0$ in the solution. Besides, the parameter $C$ is also restricted by

$$
C \geq \mp \frac{4 \sqrt{A}}{3}\left[\left(m+\frac{1}{2}\right)\left[\frac{9 \hbar^{2} A}{2 \mu}\right]^{1 / 2}+\frac{9 B}{4}\right]^{1 / 2} .
$$


So we see that the negative solution (positive in the above equation) introduces a limit on the number of bound states. The positive case (negative above) is such that we only need impose that $C$ is bigger than the left hand side of Eq. (17) with $m=0$

After this brief analysis of the region of validity of the potential parameters, we present the corresponding wave functions:

$$
\begin{aligned}
\psi_{n, m}^{\mathrm{II}}(x, y)= & {\left[\frac{32 \mu D}{\hbar^{2}}\right]^{1 / 8}\left[\left.\frac{3 \Gamma(n+1)}{2^{m+1} m ! \Gamma(a+n+1)}\right|^{1 / 2} \mid\left[\left.\frac{2 \mu D}{\hbar^{2}}\right|^{1 / 2}\left(x^{2}+y^{2}\right)\right]^{a / 2}\left[\left(\left.\arctan \frac{y}{x}\right|^{2 / 3} \beta^{2}\right]^{1 / 4}\right.\right.} \\
& \left.\times L_{n}^{a}\left[\left(\frac{2 \mu D}{\hbar^{2}}\right]^{1 / 2}\left(x^{2}+y^{2}\right)\right] H_{m}\left\{\beta \mid\left(\arctan \frac{y}{x}\right]^{2 / 3}-\frac{K}{2 A}\right]\right\} \\
& \times \exp \left\{-\left(\frac{\mu D}{2 \hbar^{2}}\right]^{1 / 2}\left(x^{2}+y^{2}\right)-\frac{\beta^{2}}{2} \mid\left[\arctan \frac{y}{x}\right]^{2 / 3}-\frac{K}{2 A}\right\}
\end{aligned}
$$

where

$$
a \equiv\left(\frac{\mu}{2 \hbar^{2} D}\right)^{1 / 2} \mathscr{E}_{n, m}^{\mathrm{II}}-(2 n+1)
$$

and

$$
\beta \equiv\left(\frac{9 \mu A}{2 \hbar^{2}}\right)^{1 / 4}
$$

This finishes the presentation of examples of CES potentials, now in two-dimensional space. The principal feature presented by these potentials, besides belonging to the CES class of potentials, is that a stringlike singularity of the potential appears. This is corroborated by the fact that the probability of finding the particle over this singularity is zero. This accounts for the interest in doing further investigations of the characteristics of such poten- tials. It would be interesting to study the behavior of their corresponding coherent states, and also see what happens with wave packets around the linear singularity of the potential.

Furthermore, in analogy with what has been done with the exact potentials $[12,13]$, and also with the quasiexact ones $[8,9]$, it is our intention to look for the dynamical algebra behind the solvability of the CES potentials. This study is presently under development and we hope to report on it in the near future.

One of the authors (A.S.D.) thanks CNPq (Conselho Nacional de Desenvolvimento Científico e Tecnológico) of Brazil for partial financial support and FAPESP (Fundação de Amparo à Pesquisa no Estado de São Paulo) for providing computer facilities.
[1] A. de Souza Dutra, Phys. Rev. A 47, R2435 (1993).

[2] E. Papp, Phys. Lett. A 178, 231 (1993).

[3] F. H. Stillinger, J. Math. Phys. 20, 1891 (1979).

[4] G. P. Flessas, Phys. Lett. 72A, 289 (1979); 78A, 19 (1980);

81A, 17 (1981); J. Phys. A 14, L209 (1981).

[5] P. G. L. Leach, J. Math. Phys. 25, 974 (1984); Physica D 17, 331 (1985).

[6] A. de Souza Dutra, Phys. Lett. A 142, 319 (1988).

[7] R. S. Kaushal, Phys. Lett. A 142, 57 (1989).

[8] M. A. Shiffman, Int. J. Mod. Phys. A 4, 2897 (1989).
[9] A. de Souza Dutra and H. Boschi Filho, Phys. Rev. A 44, 4721 (1991).

[10] L. D. Salem and R. Montemayor, Phys. Rev. A 43, 1169 (1991).

[11] T. Eguchi, P. B. Gilkey, and A. J. Hanson, Phys. Rep. 66, 213 (1980).

[12] A. O. Barut, A. Inomata, and R. Wilson, J. Phys. A 20, 4075 (1987); 20, 4083 (1987).

[13] H. Boschi-Filho and A. N. Vaidya, Ann. Phys. (NY) 212, 1 (1991). 


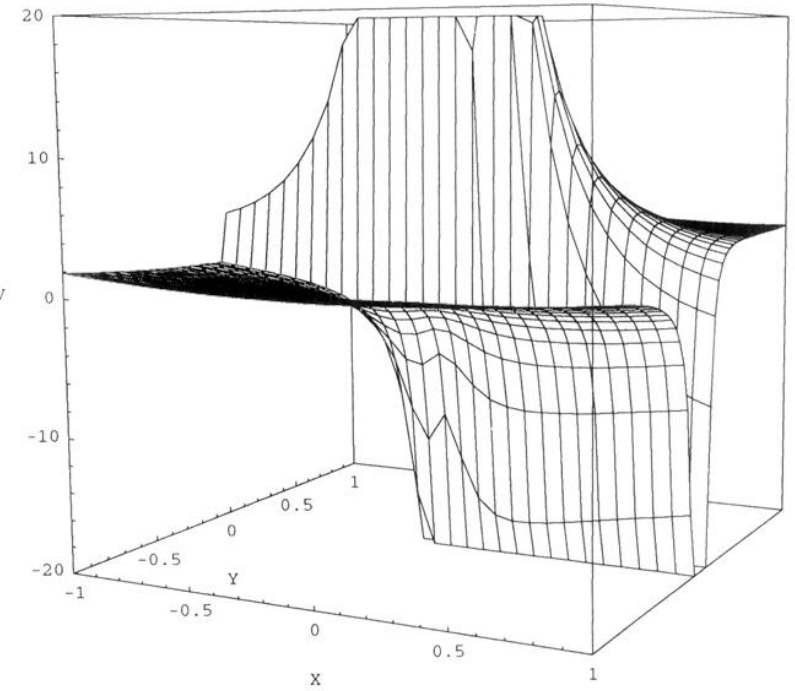

FIG. 1. Plot of the potential CES II with unitary parameters. 